\title{
Un trichobézoard dans l'estomac d'une fillette de 3 ans et 9 mois, enlevé par gastrotomie.
}

\author{
Par le Professeur S. MONRAD.
}

Au mois de mars cette année on présenta une fillette de 3 ans et 9 mois dans mon service de consultation. Pendant les deux dernières années elle avait maigri et pâli, elle n'avait plus d'appétit et était devenue fatiguée et irritable. Elle avait en outre un strophulus tenace qui durant un an avait été traité par un spécialiste des maladies de la peau. Ses parents et leur deux autres enfants étaient en bonne santé et en dehors de la maladie actuelle elle n'avait jamais eu de maladies importantes ou graves. Quand au premier examen je trouvai une grande tumeur, ferme et transversale, dans son abdomen, je la fis entrer à l'Hôpital des Enfants, en supposant qu'il s'agit d'un "gâteau abdominal", d'une péritonite tuberculeuse.

La petite était maigre et pâle, elle pesait $11 \mathrm{~kg}$. $200 \mathrm{gr}$. et avait 50 d'hémoglobine (SAHLI et TALLQUIST). L'appétit était mauvais mais elle ne vomissait pas et n'avait al solument aucune douleur. Les selles étaient souvent liquides avec mucosités. Pas de température, pas de toux, ni sueurs nocturnes. Poumons et coeur normaux. Urine normale. Dans la partie susombilicale de l'abdomen se trouvait une tumeur ferme et oblongue, dont les dimensions, la forme et le siège sont marqués sur la figure 1. Pas d'infiltrations noueuses, ni de ganglions, pas d'ascite et pas de tuméfaction du foie, ni de la rate. 
Bien que les épreuves, et de Pirquet et de Moro, par la. tuberculine plusieurs fois répétées, aussi bien avec de la tuberculine humaine que bovine, fussent négatives, je croyai cependant au début que la tumeur était un épaississement du grand épiploon de nature tuberculeuse, d'autant plus que déjà antérieurement j'avais vu les épreuves par la tuberculine

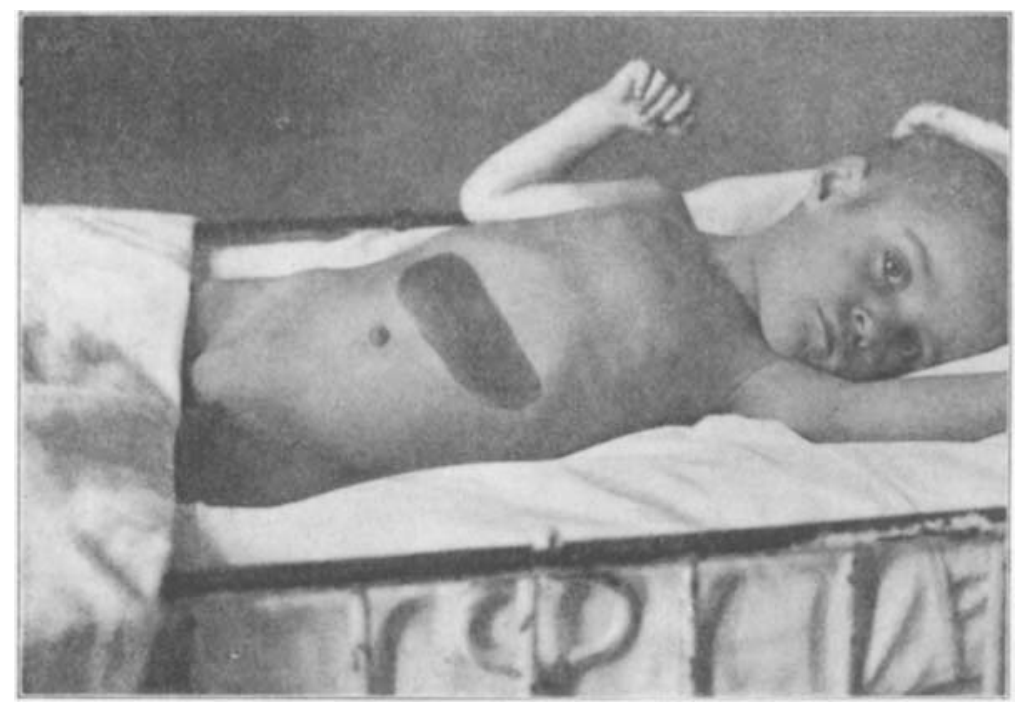

Fig. 1.

faire défaut dans la forme sèche de la péritonite tuberculeuse. L'observation ultérieure de l'enfant donna bientôt une toute autre direction à notre pensée.

Il se montra en effet que l'enfant mangeait de ses propres cheveux ainsi que de la laine des couvertures de son lit et que les selles contenaient d'assez grandes quantités de cheveux et de laine. Cependant ce n'est qu'après qu'elle eut vomi un jour une grosse masse de laine rouge (environ $70 \mathrm{gr}$ ) que nous avons pensé aux boules de cheveux qu'on trouve quelquefois dans l'estomac de l'homme et de certains ruminants. A l'examen sous anesthésie nous avons remarqué que la tumeur était lisse et qu'elle correspondait exactement à la 
forme et an siège de l'estomac. Par l'insufflation il fut constaté que la tumeur se trouvait bien dans l'estomac même.

Il était maintenant clair qu'il s'agissait d'un trichobézoord dans l'estomac, et en interrogeant la mère nous avous su que l'enfant depuis sa première année avait avalé des quantités de laine provenant d'un tapis de laine rouge sur lequel elle jouait, ainsi que pendant la dernière année elle avait avec avidité avalé de ses propres cheveux.

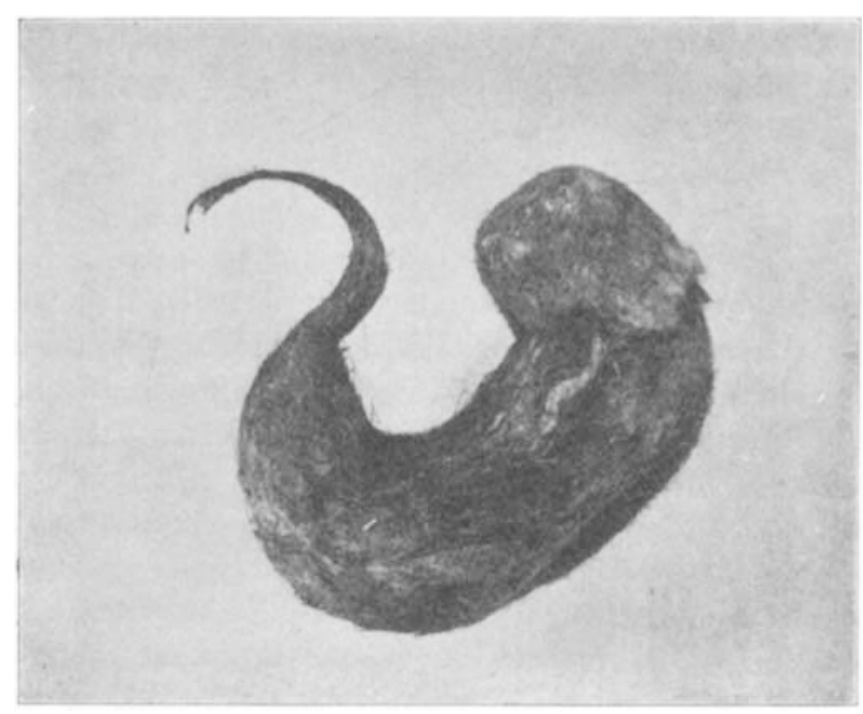

Fig. 2.

Une fois le diagnostic bien précisé, il fut fait une gastrotomie le ${ }^{20 / 4}$ (par le Docteur $\mathrm{J}_{\mathrm{OHAN}} \mathrm{U}_{\mathrm{LRICH}}$ ) et un gros trichobézoard fut enlevé. Celui-ci ne remplissait pas seulement l'estomac mais était muni d'une longue queue, qui se prolongeait assez loin dans le duodénum (voir fig. 2). La tumeur consistait surtout de cheveux et de laine du tapis et des couvertures du lit; on trouva aussi quelques fibres végétales qui semblaient provenir d'un tapis de fibres de coco.

L'état de l'opérée fut bon, et les crins furent enlevés 10 jours après. L'enfant sortit le 26 mai. 
Il va de soi qu'aussitôt que nous avions découvert ses appétits anormanx, les cheveux de l'enfant furent coupés ras, les couvertures furent enfermées dans des draps, et elle fut gardée à vue, mais nous n'avons cependant pas réussi à supprimer ses envies hystériques. Ainsi, déjà une quinzaine de jours après l'opération elle trouvait en un moment d'inattention le moyen de manger les cheveux d'une de ses poupées, comme aussi nous l'avons trouvée un autre jour très occupée à manger les ongles de ses orteils.

$\Pi$ s'agit done ici d'un cas très grave d'hystérie infantile qu'il faudra certainement beaucoup de temps pour guérir.

Tandis qu'aux autopsies dans les asiles des aliénés et pareils établissements on trouve assez souvent des tricho- et phytobézoards dans l'estomac et dans l'intestin, les cas par contre où le diagnostic a été fait sur le vivant sont encore très rares. Dans la littérature j'ai trouvé en tout 17 cas et ce n'est que chez 3 malades que le diagnostic fut posé avant l'opération. Dans les autres 14 cas on avait considéré la tumeur comme une tuberculose ou une sarcome de l'abdomen, comme une tumeur du rein ou de la rate ou comme un rein mobile, et e'est seulement à l'opération qu'on s'est aperçu qu'il s'agissait d'un trichobézoard.

Le premier cas est communiqué en 1883 par SchönborN et concerne une jeune fille de 15 ans, chlorotique, qui vers l'âge de 11 à 12 ans mangeait des cheveux ppour se faire une belle voix\%. La tumeur découverte vers l'âge de 13 ans 1/2 fut considérée comme un rein flottant et ce n'est qu'à l'opération quion trouva que c'était une boule de chevenx. Le second cas fut communiqué l'année suivante par Thornos et concerne une jeune fille de 18 ans qui pendant plusieurs années avait avalé de ses peignures et des bouts de fils coupées avec les dents "pour nettoyer la langue». Le diagnostic qui parut le plus exact fut: tumeur maligne de l'abdomen, 
mais avec le cas de Schönbors présent à l'esprit, on soupçonnait cependant l'existence d'un trichobézoard.

Le premier cas où le diagnostic fut posé avec certitude avant l'opération est communiqué par STrtazke en 1896 et concerne une jeune fille de 18 ans qui, à l'âge de croissance, avait eu l'habitude d'avaler de ses propres cheveux. Bien que les cheveux de la jeune fille fussent très blonds, les cheveux $d u$ trichobézoard étaient noirs, ce qui fut attribué à ce qu'elle avait pris du nitrate d'argent pendant plusieurs années pour des symptômes dyspeptiques.

Des 17 cas publiés jusqu'aujourd'hui, et qui tous appartiennent au sexe féminin, il y a 5 cas d'enfants. Le premier de ceux-ci est communiqué par Sснорғ et concerne une fillette de 12 ans qui depuis l'âge de 5 ans avait avalé de ses propres cheveux roux, ainsi que des poils noirs de son chien. La tumeur fut considérée comme une tumeur de la rate; c'était en réalité un trichobézoard avec une queue de $40 \mathrm{~cm}$. qui se prolongreait jusque dans le jéjunum. Le second cas fut observé par J AcoBsor en 1900 et regarde une fillette de 11 ans qui depuis sa première enfance avait mangé les bouts de ses cheveux "parce que'elle trourait agréable de se chatouiller ainsi la gorge». Pendant quelques années avant la découverte de la tumeur elle avait souffert de cardialgie et de vomissements; la tumeur fut prise pour une tumeur du rein et sa vraie nature ne s'est révelée qu'à l'opération. Du 3:e cas, communiqué par Pator en 1903, et qui touche une fillette de 9 ans, je n'ai pas pu tronver de détails. Le 4:e cas, qui date de 1908, est donné par STrLL; il s'agit d'une fillette de 9 ans qui mangeait ses cheveux depuis l'âge de 3 ans. La tumeur, découverte 7 mois avant l'opération, était considérée comme une lymphosarcome. Le ò:e cas, publié en 1908 par Harvie, concerne une fillette de 9 ans qui depuis sa première année mangeait des cheveux et de la laine; pendant plusieurs années elle avait souffert de cardialgie, nausées et vomissements et on avait souvent trouvé des cheveux dans ses selles. Le diagnostic exact fut posé avant l'opération.

Notre cas, qui devient ainsi le 6:e dans la série des tri- 
chobézoards trouvés dans l'estomac de l'enfant, est d'autant plus digne d'intérêt que l'enfant n'avait pas encore 4 ans quand le diagnostic fut posé et la boule de cheveux enlevée.

En parcourant les cas publiés on voit que les symptômes subjectifs sont très imprécis; le plus souvent il s'agit d'anorexie, de cardialgie et de vomissements, mais souvent il n'y a pas de phénomènes dyspeptiques et seulement de vagues symptômes de chlorose et de troubles nerreux. Dans les cas où l'examen de la fonction stomacale a été fait, on a trouvé la force digestive de l'estomac normale. Objectivement on trouve une grosseur dans la partie susombilicale de l'abdomen - en déterminer le siège et la nature c'est poser le diagnostic. Il fant noter ici que plusieurs des malades chez qui une grosse tumeur a été découverte depuis longtemps ne s'en trourent que très peu incommodés, ce qui n'est évidemment pas farorable à l'hypothèse d'une tumeur maligne. Mais ce qui est d'importance essentielle, c'est l'observation des goûts anormaux de la malade, le fait de trouver dans ses selles des cheveux, de la laine et pareilles choses, et la constatation que la tumeur se trouve dans l'estomac même.

Aussitôt que le diagnostic est posé, la tumeur doit être enlevée par la gastrotomie, et cette opération a réussi dans tous les cas publiés jusque maintenant. Mais il faut en suite à tout prix combattre les goûts hystériques de la malade, sans quoi on risque de voir se former petit à petit un nouveau trichobézoard.

Que ces tumeurs ne sont certes pas tout à fait inoffensives démontre entre autres un cas publié par BREwsTer, qui en 1900 opéra une fillette de 10 ans pour iléus et qui en trouva la cause dans un trichobézoard logé dans l'intestin grêle. La fillette qui pendant plusieurs années avait absorbé des quantités de ses cheveux était si affaiblie avant l'opération même, qu'elle succomba peu de temps après. 